\title{
Correlating Chemical Sensitivity with Low Level Activation of Mechanotransduction Pathways in Hematologic Malignancies
}

\author{
Robert G. Hawley*๑ \\ Department of Anatomy and Regenerative Biology, School of Medicine and Health Sciences, \\ George Washington University, Washington, DC 20037, USA
}

\begin{abstract}
Large-scale screening has revealed that human hematopoietic cancer cell lines are generally more sensitive to various classes of drugs than cell lines established from solid tumors. A detailed examination of data in the Cancer Therapeutics Response Portal (http://portals.broadinstitute.org/ctrp/) suggests that this enhanced sensitivity is due to lower basal levels of activation of TAZ-TEAD mechanotransduction pathways in hematopoietic versus non-hematopoietic cells. Translation inhibitors such as omacetaxine mepesuccinate (homoharringtonine) fall into this category of hematopoietic-selective compounds. Moreover, additional molecular determinants of sensitivity suggest that homoharringtonine might show therapeutic efficacy in certain patients with advanced hematologic malignancies despite activation of these pathways.
\end{abstract}

In a recent publication, Rees et al. ${ }^{1}$ reported a new computational tool capable of elucidating small molecule mechanisms of action by correlating the sensitivity patterns of 481 compounds with 18,543 basal transcript levels across a large panel of human cancer cell lines (CCLs) and identifying differential gene expression patterns characterized by specific outlier transcripts. Overall, they measured the responses of 823 unique CCLs out of 947 previously characterized as part of the Cancer Cell Line Encyclopedia (CCLE) project. ${ }^{2}$ The data and correlation methods have been made publicly available through the Cancer Therapeutics Response Portal (CTRP; http://portals.broadinstitute.org/ctrp/). ${ }^{1}$

The authors indicated that hematopoietic CCLs were generally more sensitive to many small molecule perturbagens than were CCLs from solid tumors and they observed that outlier transcripts corresponding to putative biomarker genes for hematopoietic CCLs showed significant correlation with many compounds. ${ }^{1}$ They speculated that these correlations reflected the general sensitivity of hematopoietic CCLs, rather than a mechanistic connection

Keywords: TAZ-TEAD; Omacetaxine mepesuccinate (homoharringtonine); RPL3, TNFRSF $12 A$.

Abbreviations: CCLE, Cancer Cell Line Encyclopedia; CCLs, cancer cell lines; CTRP, Cancer Therapeutics Response Portal; $r$, Pearson expression-sensitivity correlation coefficient; TAZ, transcriptional co-activator with PDZ-binding motif; TEAD1, TEA domain transcription factor 1; TJP1, tight junction protein 1 (also known as zonula occludens 1); $z$, Fisher's $z(r)$-scored Pearson expression-sensitivity correlation coefficient.

Received: July 12, 2017; Revised: August 28, 2017; Accepted: August 30, 2017

*Correspondence to: Robert G. Hawley, Department of Anatomy and Regenerative Biology, School of Medicine and Health Sciences, George Washington University, 2300 I Street NW, Washington, DC 20037, USA. E-mail: rghawley@gwu.edu

How to cite this article: Hawley RG. Correlating Chemical Sensitivity with Low Level Activation of Mechanotransduction Pathways in Hematologic Malignancies. Explor atory Research and Hypothesis in Medicine 2017;2(3):63-67. doi: 10.14218/ERHM. 2017.00022 . of biomarker genes to compounds

In particular, Rees et al. ${ }^{1}$ noted that an outlier transcript, TNFRSF 12A, was the transcript most correlated with sensitivity to 36 small molecules enriched for several classes of drugs - including microtubule modulators, chromatin modifiers, topoisomerase inhibitors and nucleotide analogs - where low expression correlated with hematopoietic CCL sensitivity $(z$ scores $>15.857$; where $r$ is the Pearson expression-sensitivity correlation coefficient and $z$ is Fisher's $z(r)$-scored Pearson expression-sensitivity correlation coefficient).

We recently demonstrated that activation of interacting transcriptional effectors of the Hippo signaling cascade, transcriptional co-activator with PDZ-binding motif (TAZ) encoded by the WWTR1 gene and TEA domain transcription factor 1 (TEAD1) involved in the transduction of cytoskeletal, adhesive and mechanical cues, ${ }^{3}$ contributed to the proteasome inhibitor-resistant phenotype of a multiple myeloma cell line. ${ }^{4}$ This was associated with elevated expression of TAZ-TEAD1 target genes (e.g., ITGB5 and CRIMI), ${ }^{5}$ as well as the tight junction protein gene TJPI (also known as zonula occludens $1, \mathrm{ZO}-1)^{4}$

This molecular phenotype was shared by a subset of multiple myeloma patients who developed relapsed/refractory disease while on proteasome inhibitor-based therapy. Additionally, Connectivity Map analysis predicted that translation inhibitors may overcome the drug-resistant state; ${ }^{6,7}$ we confirmed this prediction by showing that omacetaxine mepesuccinate (homoharringtonine) - the first translation inhibitor to be approved by the United States Food and Drug Administration-displayed potent cytotoxicity on the proteasome inhibitor-resistant multiple myeloma cells. ${ }^{4,8}$

In an attempt to obtain a generalizable biomarker of homoharringtonine sensitivity, I queried the CTRP using the CellMinerCDB web application (https://discover.nci.nih.gov/cellminer$\mathrm{cdb} /)^{9}$ The RPL3 gene encoding ribosomal protein L3 of the large 

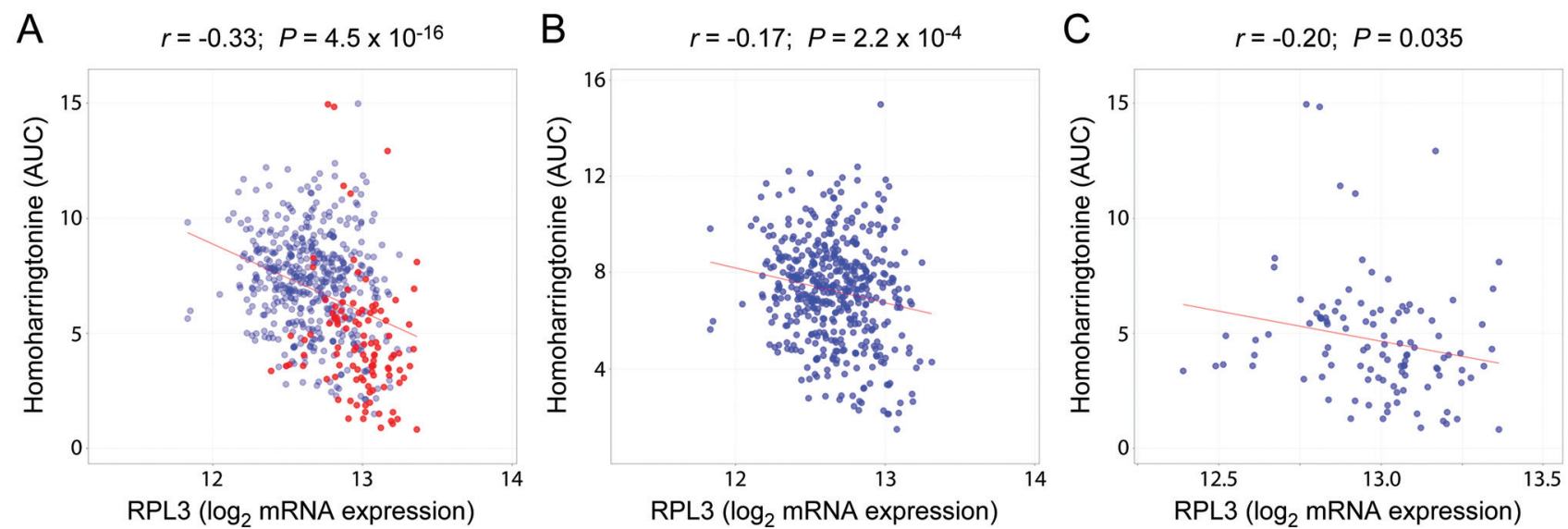

Fig. 1. Human CCLs with increased RPL3 expression exhibit enhanced sensitivity to the translation inhibitor omacetaxine mepesuccinate (homoharringtonine). A. Expression-sensitivity correlations for homoharringtonine and RPL3 expression for hematopoietic and non-hematopoietic CCLs in the CTRP. Hematopoietic CCLs are highlighted in red. B. Expression-sensitivity correlations for homoharringtonine and RPL3 expression for non-hematopoietic CCLs in the CTRP. C. Expression-sensitivity correlations for homoharringtonine and RPL3 expression for hematopoietic CCLs in the CTRP. Expression-sensitivity correlations were determined using the CellMinerCDB web application. ${ }^{9}$ AUC, area under the concentration-response curve; $r$, Pearson expression-sensitivity correlation coefficient.

60S ribosomal subunit bound by homoharringtonine was identified as a potential candidate (https://www.drugbank.ca/drugs/ DB04865\#targets); 10,11 both hematopoietic and non-hematopoietic CCLs with increased RPL3 expression exhibit enhanced sensitivity to the drug (Fig. 1). This is an attractive possibility for further evaluation since up-regulation of ribosome biogenesis is frequently associated with tumor progression and adverse clinical outcomes. ${ }^{12,13}$

Unexpectedly, I also discovered that low TNFRSF12A expression correlated with sensitivity to homoharringtonine $\left(93^{\text {rd }}\right.$ ranked compound out of $481 ; z$ score $=12.47$ ) (Fig. 2A). Additionally, I found that low TNFRSF12A expression correlated with sensitivity to another translation inhibitor, the silvestrol analog CR-1-31B $\left(45^{\text {th }}\right.$ ranked compound out of $481 ; z$ score $\left.=15.46\right)$ which inhibits cap-dependent translation initiation by targeting the eIF4A RNA helicase (Fig. 2B). ${ }^{14,15}$ I subsequently learned that TNFRSF12A is a direct target of TEAD transcription factors complexed with
TAZ or its paralog YAP $1 .{ }^{16}$ Notably, TNFRSF $12 A$ is coexpressed (top 20 gene neighbors) with WWTR1/TAZ, YAP1, TEAD1, ITGB5, CRIM1 and TJP1 as well as other YAP/TAZ direct targets (e.g., CYR61) ${ }^{16}$ across all CCLE CCLs (Fig. S1).

Hematopoietic CCLs generally expressed lower levels of these transcripts than non-hematopoietic CCLs (Fig. 3, Fig. S1). Furthermore, DAVID analysis of the overlapping outlier transcripts for homoharringtonine and CR-1-31B sensitivity (homoharringtonine, $z$ score $>9$; CR-1-31B, $z$ score $>11$ ) (Fig. 4) revealed that the most significantly enriched cluster comprises YAP/TAZ annotation terms (Table S1) ${ }^{17}$ whereas oPOSSUM-3 transcription factor binding site motif discovery identified TEAD1 as the top-ranked overrepresented motif in the set of overlapping genes (Table S1). ${ }^{18}$

Further inspection of the list of 481 compounds in the CTRP uncovered a third translation inhibitor, the silvestrol analog, SR-II138A. It turned out that SR-II-138A is among the 36 compounds most highly associated with low TNFRSF12A expression $\left(24^{\text {th }}\right.$
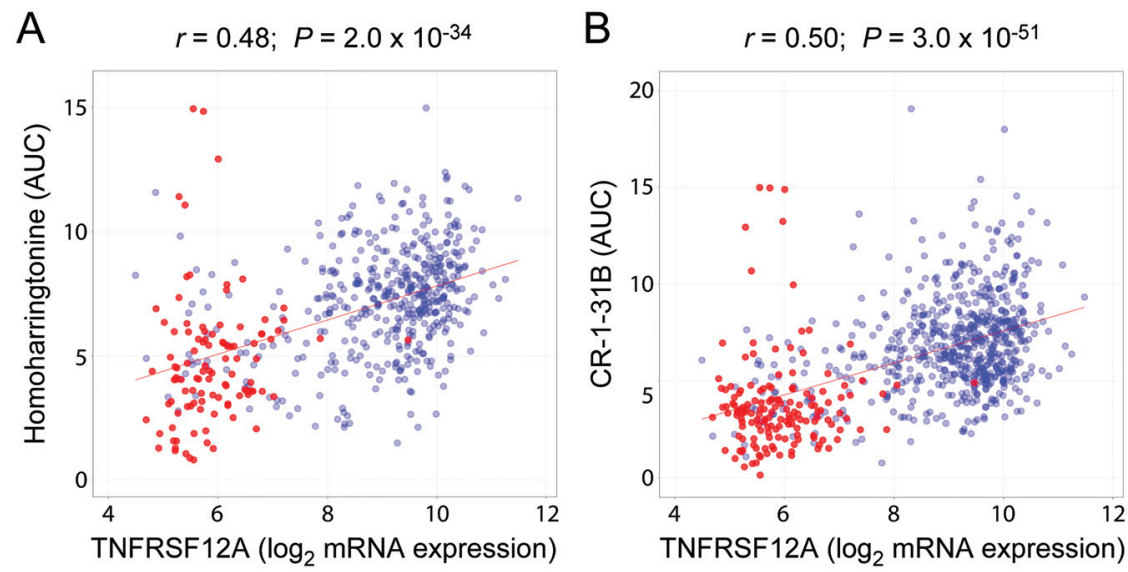

Fig. 2. Human CCLs with low level TNFRSF12A expression exhibit enhanced sensitivity to the translation inhibitors omacetaxine mepesuccinate (homoharringtonine) and CR-1-31B. A. Expression-sensitivity correlations for homoharringtonine and TNFRSF12A expression for hematopoietic and non-hematopoietic CCLs in the CTRP. Hematopoietic CCLs are highlighted in red. B. Expression-sensitivity correlations for CR-1-31B and TNFRSF12A expression for hematopoietic and non-hematopoietic CCLs in the CTRP. Hematopoietic CCLs are highlighted in red. Expression-sensitivity correlations were determined using the CellMinerCDB web application. ${ }^{9} \mathrm{AUC}$, area under the concentration-response curve; $r$, Pearson expression-sensitivity correlation coefficient. 


\section{TNFRSF12A}
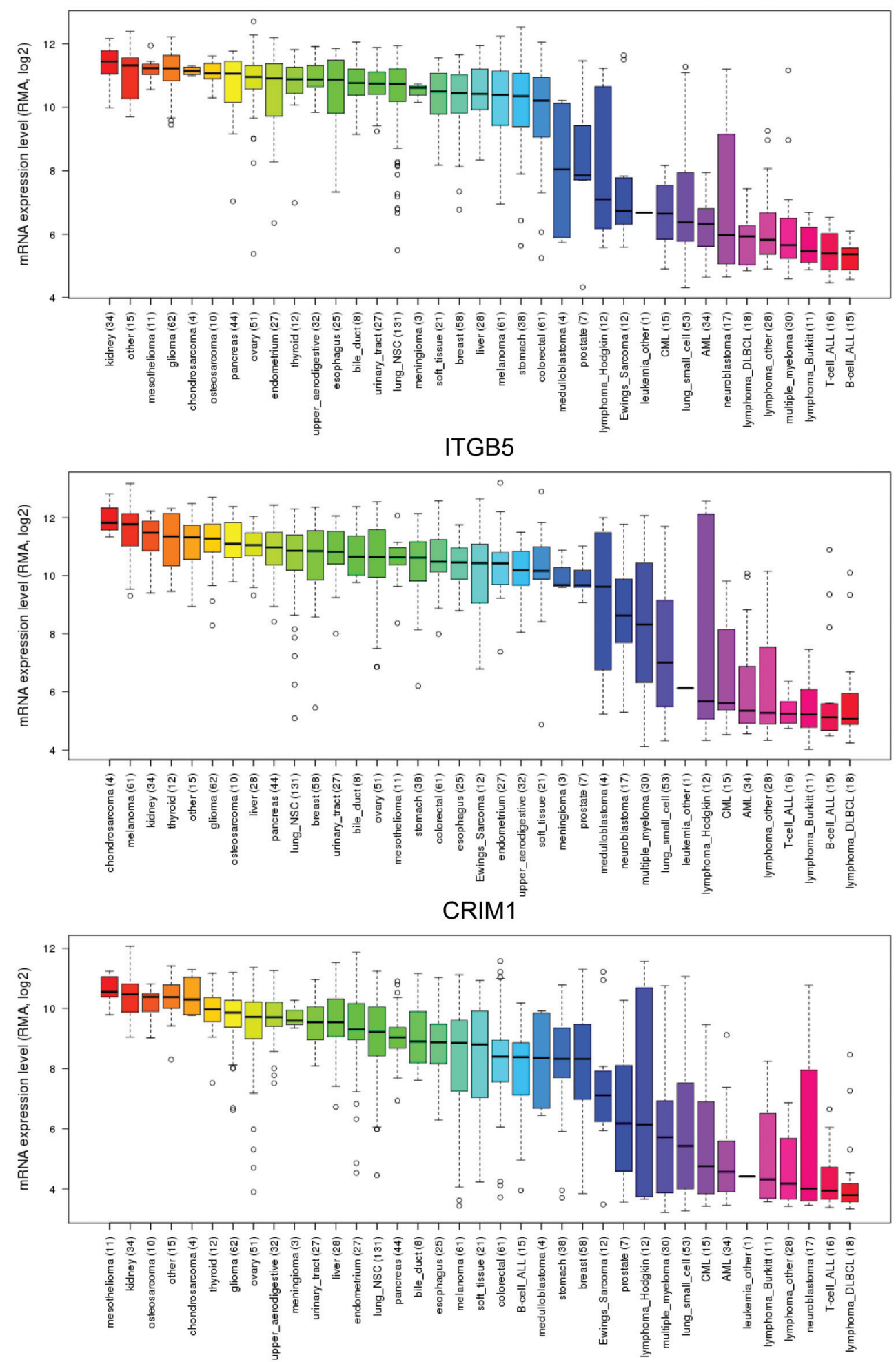

Fig. 3. Relative expression levels of TNFRSF12A, ITGB5 and CRIM1 in hematopoietic and non-hematopoietic CCLs in the CCLE. Box-and-whisker plots show the transcript distribution levels for each lineage, ordered by the median expression level (line), the inter-quartile range (box) and up to $1.5 \times$ the inter-quartile range (bars). ${ }^{2}$ Sample numbers $(n)$ are indicated in parentheses. Note that some non-hematopoietic CCLs that were observed to be sensitive to many of the small molecule perturbagens to which hematopoietic CCLs are sensitive (e.g., CCLs from the neuroblastoma lineage ${ }^{1}$ ), also generally express lower levels of these transcripts. RMA, robust-multi-array-average values. 

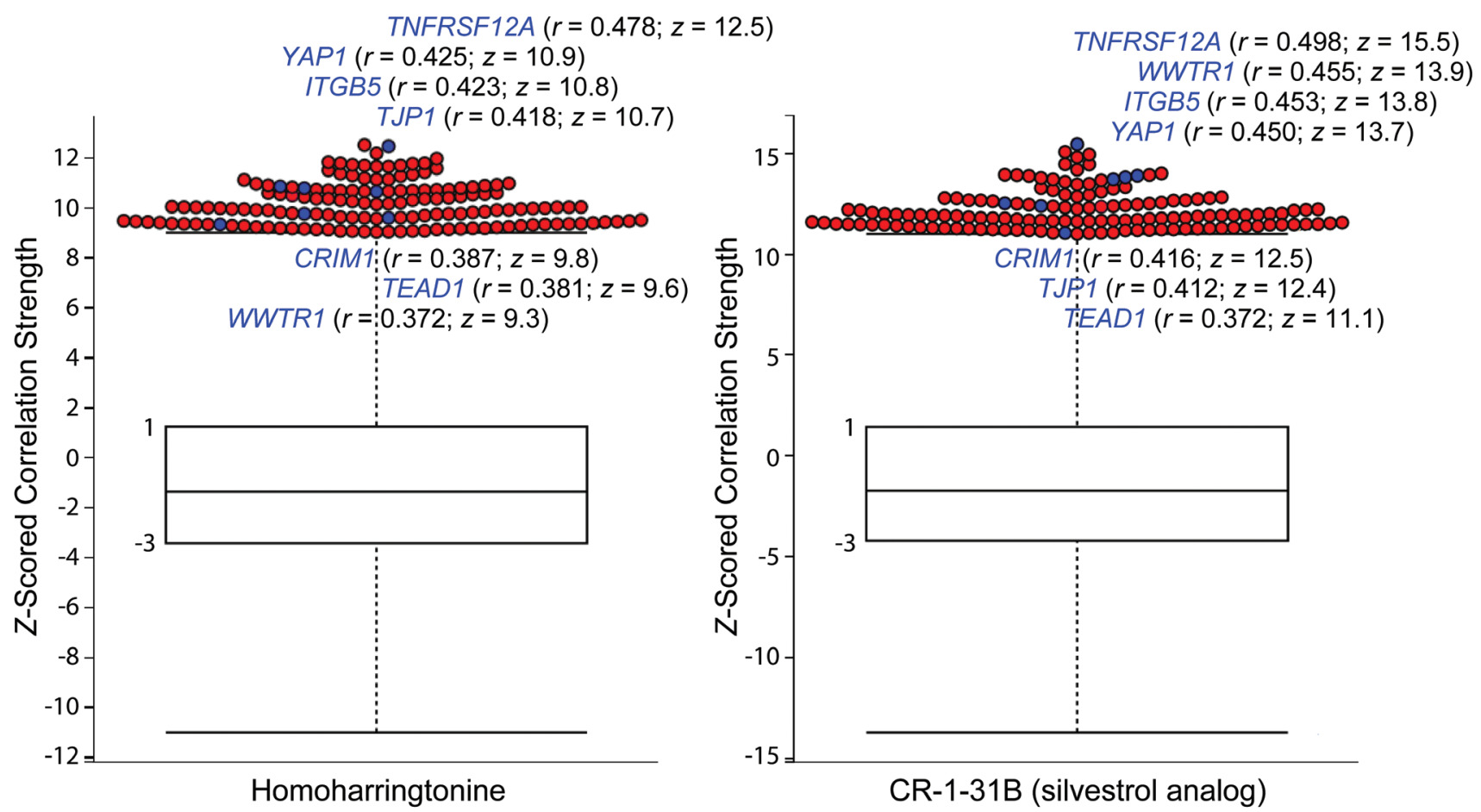

Fig. 4. Box-and-whisker plots of 18,543 correlation coefficients of transcript levels to omacetaxine mepesuccinate (homoharringtonine; left) and CR-131B (right) sensitivity. Expression-sensitivity correlations were determined across all CCLs (any primary site/subtype and any growth mode) using the CTRP resource. ${ }^{1}$ Outlier points represent 144 transcripts for homoharringtonine $(z$ score $>9)$ and 138 transcripts for CR-1-31B $(z$ score $>11)$. TNFRSF12A is highlighted as are YAP1, WWTR1/TAZ, TEAD1, TJP1, ITGB5 and CRIM1. $r$, Pearson expression-sensitivity correlation coefficient. $z, z(r)$-scored Pearson expressionsensitivity correlation coefficient. See Table S1 for the complete lists of outlier transcripts.

ranked compound out of $481 ; z$ score $=16.56)($ see Supplementary Data Set 7 of Rees et al. ${ }^{1}$ ). I interpret these observations to indicate that the generally increased sensitivity of hematopoietic CCLs to several classes of drugs compared to non-hematopoietic CCLs is due to lower basal levels of activation of TAZ-TEAD mechanotransduction pathways. ${ }^{3,19}$ Translation inhibitors, which appear to be a class of drugs that can be included in this category, may thus demonstrate therapeutic efficacy against hematologic malignancies, in front-line as well as in certain relapsed/refractory settings. ${ }^{4,8}$

\section{Acknowledgments}

I thank Irene Riz for critical comments on the manuscript. The author gratefully acknowledges financial support from the Katzen Cancer Research Center at the GW Cancer Center and a generous philanthropic gift from Marc Cohen. RGH was supported in part by NIH grant R21AI113806.

\section{Conflict of interest}

The author has no conflict of interest related to this publication.

Author contributions

Manuscript writing (RGH).

\section{Supporting information}

Supplementary material for this article is available at https://doi. org/10.14218/ERHM.2017.00022.

Fig. S1. Heat maps of the top 20 neighbors of TNFRSF12A, YAP1, WWTR1/TAZ, TEAD1, TJP1, ITGB5 and CRIM1 across all CCLs in the CCLE. ${ }^{2}$ Dark red indicates the highest expression levels. Dark blue indicates the lowest expression levels. TNFRS$F 12 A$ is indicated with a red asterisk and YAP1, WWTR1/TAZ, TEAD1, TJP1, ITGB5 and CRIM1 are indicated with blue asterisks on the right side of the figure. Note that the expression levels of TNFRSF12A, YAP1, WWTR1/TAZ, TEAD1, TJP1, ITGB5 and CRIM1 are generally lower in hematopoietic CCLs (samples labeled "HAEMATOPOIETIC_AND_LYMPHOID_TISSUE") than in non-hematopoietic CCLs.

Table S1. The complete lists of outlier transcripts for homoharringtonine and $C R-1-31 B$ sensitivity.

\section{References}

[1] Rees MG, Seashore-Ludlow B, Cheah JH, Adams DJ, Price EV, Gill $\mathrm{S}$, et al. Correlating chemical sensitivity and basal gene expression reveals mechanism of action. Nat Chem Biol 2016;12(2):109-116. doi:10.1038/nchembio.1986.

[2] Barretina J, Caponigro G, Stransky N, Venkatesan K, Margolin AA, Kim $\mathrm{S}$, et al. The Cancer Cell Line Encyclopedia enables predictive modelling of anticancer drug sensitivity. Nature 2012;483(7391):603-607. doi:10.1038/nature11003. 
[3] Dupont S. Role of YAP/TAZ in cell-matrix adhesion-mediated signalling and mechanotransduction. Exp Cell Res 2016;343(1):42-53. doi:10.1016/j.yexcr.2015.10.034.

[4] Riz I, Hawley RG. Increased expression of the tight junction protein TJP1/ZO-1 is associated with upregulation of TAZ-TEAD activity and an adult tissue stem cell signature in carfilzomib-resistant multiple myeloma cells and high-risk multiple myeloma patients. Oncoscience 2017;Aug 1. doi: 10.18632/oncoscience.356. [Epub ahead of print].

[5] Cordenonsi M, Zanconato F, Azzolin L, Forcato M, Rosato A, Frasson $C$, et al. The Hippo transducer TAZ confers cancer stem cellrelated traits on breast cancer cells. Cell 2011;147(4):759-772. doi:10.1016/j.cell.2011.09.048.

[6] Lamb J, Crawford ED, Peck D, Modell JW, Blat IC, Wrobel MJ, et al. The Connectivity Map: using gene-expression signatures to connect smal molecules, genes, and disease. Science 2006;313(5795):1929-1935. doi:10.1126/science.1132939.

[7] Subramanian A, Narayan R, Corsello SM, Peck DD, Natoli TE, Lu X, et al. A next generation Connectivity Map: L1000 platform and the first 1,000,000 profiles. bioRxiv 2017; doi:10.1101/136168.

[8] Alvandi F, Kwitkowski VE, Ko CW, Rothmann MD, Ricci S, Saber H, et al. U.S. Food and Drug Administration approval summary: omacetaxine mepesuccinate as treatment for chronic myeloid leukemia. Oncologist 2014;19(1):94-99. doi:10.1634/theoncologist.2013-0077.

[9] Luna A, Rajapakse VN, Sousa FG, Gao J, Schultz N, Varma S, et al. rcellminer: exploring molecular profiles and drug response of the NCl-60 cell lines in R. Bioinformatics. 2016;32(8):1272-1274. doi:10.1093/bioinformatics/btv701.

[10] Law V, Knox C, Djoumbou Y, Jewison T, Guo AC, Liu Y, et al. DrugBank 4.0: shedding new light on drug metabolism. Nucleic Acids Res 2014;42(Database issue):D1091-D1097. doi:10.1093/nar/gkt1068.

[11] Fresno M, Jimenez A, Vazquez D. Inhibition of translation in eukary- otic systems by harringtonine. Eur J Biochem 1977;72(2):323-330. doi:10.1111/j.1432-1033.1977.tb11256.x.

[12] Belin S, Beghin A, Solano-Gonzalez E, Bezin L, Brunet-Manquat S, Textoris J, et al. Dysregulation of ribosome biogenesis and translational capacity is associated with tumor progression of human breast cancer cells. PLoS One 2009;4(9):e7147. doi:10.1371/journal. pone.0007147.

[13] Zhang D, Park D, Zhong Y, Lu Y, Rycaj K, Gong S, et al. Stem cell and neurogenic gene-expression profiles link prostate basal cells to aggressive prostate cancer. Nat Commun 2016;7:10798. doi:10.1038/ ncomms10798.

[14] Jin C, Rajabi H, Rodrigo CM, Porco JA Jr, Kufe D. Targeting the elF4A RNA helicase blocks translation of the MUC1-C oncoprotein. Oncogene 2013;32(17):2179-2188. doi:10.1038/onc.2012.236.

[15] Wolfe AL, Singh K, Zhong Y, Drewe P, Rajasekhar VK, Sanghvi VR, et al. RNA G-quadruplexes cause elF4A-dependent oncogene translation in cancer. Nature 2014;513(7516):65-70. doi:10.1038/nature13485.

[16] Zanconato F, Forcato M, Battilana G, Azzolin L, Quaranta E, Bodega B et al. Genome-wide association between YAP/TAZ/TEAD and AP-1 at enhancers drives oncogenic growth. Nat Cell Biol 2015;17(9):12181227. doi:10.1038/ncb3216.

[17] Huang D, Sherman BT, Lempicki RA. Systematic and integrative analysis of large gene lists using DAVID bioinformatics resources. Nat Protoc 2009;4(1):44-57. doi:10.1038/nprot.2008.211.

[18] Kwon AT, Arenillas DJ, Worsley Hunt R, Wasserman WW. oPOSSUM-3. advanced analysis of regulatory motif over-representation across genes or ChIP-Seq datasets. G3 (Bethesda) 2012;2(9):987-1002. doi:10.1534/g3.112.003202.

[19] Kim MH, Kim J. Role of YAP/TAZ transcriptional regulators in resistance to anti-cancer therapies. Cell Mol Life Sci 2017;74(8):14571474. doi:10.1007/s00018-016-2412-x. 